\title{
Lieb-Schultz-Mattis theorem for quasitopological systems
}

\author{
Michael Freedman, ${ }^{1}$ Chetan Nayak, ${ }^{1,2}$ and Kirill Shtengel ${ }^{3,4, *}$ \\ ${ }^{1}$ Microsoft Research, Station Q, CNSI Building, University of California, Santa Barbara, California 93106, USA \\ ${ }^{2}$ Department of Physics, University of California, Santa Barbara, California 93106, USA \\ ${ }^{3}$ Department of Physics and Astronomy, University of California, Riverside, California 92521, USA \\ ${ }^{4}$ California Institute of Technology, Pasadena, California 91125, USA
}

(Received 31 August 2005; revised manuscript received 5 August 2008; published 11 November 2008)

\begin{abstract}
In this paper we address the question of the existence of a spectral gap in a class of local Hamiltonians. These Hamiltonians have the following properties: their ground states are known exactly; all equal-time correlation functions of local operators are short-ranged; and correlation functions of certain nonlocal operators are critical. A variational argument shows gaplessness with $\omega \propto k^{2}$ at critical points defined by the absence of certain terms in the Hamiltonian, which is remarkable because equal-time correlation functions of local operators remain short ranged. We call such critical points, in which spatial and temporal scaling are radically different, quasitopological. When these terms are present in the Hamiltonian, the models are in gapped topological phases which are of special interest in the context of topological quantum computation.
\end{abstract}

DOI: 10.1103/PhysRevB.78.174411

PACS number(s): 75.10.Jm, 05.30.- d, 05.50.+q

\section{INTRODUCTION}

Recently, there has been great interest in various unconventional states of matter, in particular those that might appear in strongly interacting two-dimensional (2D) systems. These exotic phases may be characterized by unconventional order parameters. Alternatively, the phases may be topologi$\mathrm{cal}$. In this case there is no local order parameter, but there may be a nonlocal order parameter, which is related to the topological properties of the state: exotic braiding statistics, quantum number fractionalization, and a ground-state (GS) degeneracy which depends only on the topology of the underlying 2D manifold. ${ }^{1}$ The low-energy effective description of these phases is a topological quantum-field theory (TQFT). A variety of topological phases are known to exist in the quantum Hall systems. ${ }^{2}$ It has been conjectured that such phases also occur in frustrated magnets where they may be connected to superconductivity. ${ }^{3-9}$ Topological phases are also an attractive platform for quantum computation, where their insensitivity to local perturbations leads to fault tolerance. ${ }^{10}$ Non-Abelian topological phases are particularly interesting in this context because in many of these phases, the braiding of quasiparticles generates a set of transformations which is sufficient for universal quantum computation. ${ }^{11}$ In this paper, we shall concentrate on microscopic models which are related to classes of $P$ - and $T$-invariant non-Abelian topological phases. ${ }^{12}$

A set of conditions which places a microscopic model in such a topological phase can be briefly summarized as follows. We suppose that the low-energy Hilbert space can be mapped onto that of a quantum loop gas. This is the case in a large class of models, including dimer models, certain spin models, and some interacting hard-core boson models. In such a model, basis states are associated to collections of nonintersecting loops. ${ }^{12-14}$ We give some examples in Sec. III. A Hamiltonian can act on states in this Hilbert space by doing the following: (i) the loops can be continuously deformed-we will call this an isotopy move; (ii) a small loop can be created or annihilated-the combined effect of this move and the isotopy move has been dubbed " $d$-isotopy;" $12,14,15$ and (iii) finally, when exactly $k+1$ strands come together in some local neighborhood, the Hamiltonian can cut them and reconnect the resulting "loose ends" pairwise so that the newly formed loops are still nonintersecting. More specifically, in order for this model to be in a topological phase, the ground state of this Hamiltonian should be a superposition of all such pictures with the additional requirements that (i) if two pictures can be continuously deformed into each other, they enter the GS superposition with the same weight; (ii) the amplitude of a picture with an additional loop is $d$ times that of a picture without such loop; and (iii) this superposition is annihilated by the application of the Jones-Wenzl (JW) projector that acts locally by reconnecting $k+1$ strands (for a detailed description see the following section). The main goal of this paper is to investigate the energy spectrum of a system subject to the first two conditions.

We shall show that a generic local Hamiltonian which enforces $d$-isotopy for its ground state(s) is necessarily gapless provided that $|d| \leq \sqrt{2}$. (We shall also argue that, surprisingly, even the addition of the JW projector to the Hamiltonian will not open a gap for $|d|=\sqrt{2}$.) Such a Hamiltonian is a sum of projection operators (enforcing on every plaquette of the lattice both isotopy invariance and the value $d$ for a contractible loop). These projection operators do not commute with each other, but they are compatible with each other in the sense that they all annihilate the ground state. (Such Hamiltonians have also arisen in the context of the quantum Hall effect, where the Haldane pseudopotentials are projection operators which annihilate the Laughlin states, ${ }^{16,17}$ but do not commute so the excited states are not known exactly, and, under a general name of "parent Hamiltonians," in quantum antiferromagnetism. ${ }^{18-20}$ ) Exact knowledge of the ground state enables us to construct a variational ansatz for the lowest energy excited state. The strategy is quite similar to the single-mode approximation (SMA), in which $\rho_{q}|0\rangle$ is the trial excited state, where $\rho$ is some conserved charge. In the case of a broken-symmetry state, $\rho$ is the charge which generates the symmetry transformation which is spontane- 
ously broken in the ground state. Our method generalizes the SMA in an important way: We do not rely on the existence of any conserved charges and, indeed, the model need not have any. Instead, we have the less restrictive condition that the configuration space of the model should break into two (or more) sectors whose volume is parametrically larger than the boundary between them, e.g., by a factor of the system size $L$. If the Hamiltonian only has matrix elements between nearby points in configuration space, then (à la Lieb-Schultz-Mattis ${ }^{21}$ ) we can construct a wave function which is equal to the ground-state wave function except for a relative sign change between the two sectors. The energy cost of this "twisted" excited state would be at most $\sim 1 / L$ and is even smaller in the case of the models which we consider here. On the other hand, if the Hamiltonian directly connects the two putative sectors then it means that in reality these are not two distinct sectors, and a sign change in the wave function would have an energetic penalty which does not scale to zero as $L \rightarrow \infty$.

In this paper, we prove the general result on gaplessness described in the previous paragraph.. We apply this result to microscopic models implementing $d$-isotopy and find the remarkable result that they are gapless with $\omega \propto k^{2}$ in spite of the absence of any power-law equal-time correlation functions of local operators.

\section{II. $d$-ISOTOPY AND ITS LOCAL SUBSPACES}

In this section we present a more formal definition of $d$-isotopy; it can be skipped on a first reading. Readers interested in the details are also referred to Refs. 12, 13, and 15.

Although we will eventually be considering a system on a lattice, it is useful to begin by defining the Hilbert spaces of interest $\bar{V}_{d}$ and $V_{d}$ in the smooth lattice-free setting. Consider a compact surface $Y$ and the set $S$ of all multiloops ${ }^{22} X \subset Y$. If $\partial Y$ (the boundary of $Y$ ) is nonempty, we fix once and for all a finite set $P$ of points on $\partial Y$ with $X \cap \partial Y=P$. We assume $Y$ is oriented but $X$ should not be. There is a large vector space $\mathrm{C}^{S}$ of complex-valued functions on $S$. We say $X$ and $X^{\prime}$ are isotopic $\left(X \sim X^{\prime}\right)$ if one may be gradually deformed into the other with, of course, the deformation being the identity on $\partial Y$ (see Fig. 1).
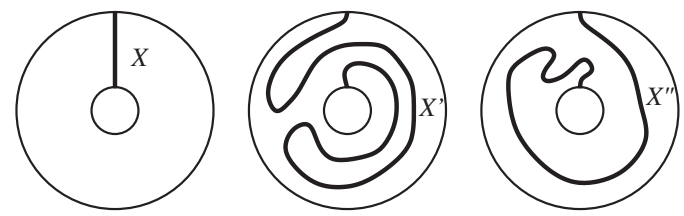

FIG. 1. Isotopy on an annulus: $X \sim X^{\prime}$ but $X \nsim X^{\prime \prime}$.

We may view the isotopy relation as a family of linear constraints on $\mathrm{C}^{S}$, namely, $\Psi(X)-\Psi\left(X^{\prime}\right)=0$ if $X \sim X^{\prime}$. The subspace satisfying these linear constraints is now only of countable dimension; it consists of those functions which depend only of the isotopy class $[X]$ of $X$ and may be identified with $C^{[S]}$, where $[S]$ is the isotopy classes of multiloops with fixed boundary conditions. Note that all isotopes can be made by composing small locally supported ones so the relations we just imposed are "local," in the sense that they can be implemented with purely local terms in the Hamiltonian.

Let us go further and define an additional local relation which when added to isotopy constitutes the " $d$-isotopy" relation. This relation is

$$
d \Psi(X)-\Psi(X \cup \bigcirc)=0 .
$$

It says that if two multiloops are identical except for the presence of a small (or, it follows, any contractible) circle then their function values differ by a factor of $d$ : a fixed positive real number. In cases of interest to us $1 \leq d<2$, so our function is either neutral to or "likes" small circles. We call the subspace obeying all these constraints the $d$-isotopy space of $Y$ (with fixed boundary conditions) and write it as $\bar{V}_{d} \subset \mathrm{C}^{[S]} \subset \mathrm{C}^{S}$. The subspace $\bar{V}_{d}\left(T^{2}\right)$ is still of countable dimension or extensively degenerate on the torus $T^{2}$. The vector space $\bar{V}_{d}$ is clearly the ground-state manifold (GSM) of a local Hamiltonian acting on $\mathrm{C}^{[S]}$.

It is a remarkable fact ${ }^{12,15}$ that it is very difficult to add any further local relations to $d$-isotopy without killing the vector space entirely. For the physically interesting cases $\alpha$ $=\mathrm{e}^{\pi i /(k+2)}$ and $k=1,2,3, \ldots$, there is such a local relation and a natural positive-definite inner product on $\bar{V}_{d}$ (see Refs. 12 and 15 for definition).

In these cases the local relations are essentially the JonesWenzl idempotents,

$k=1$ :

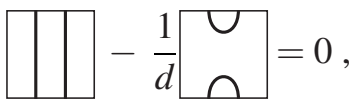

$k=2$ :

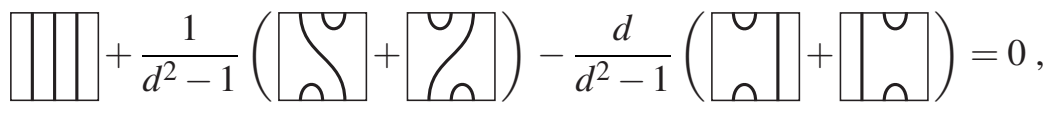


$k=3$ :

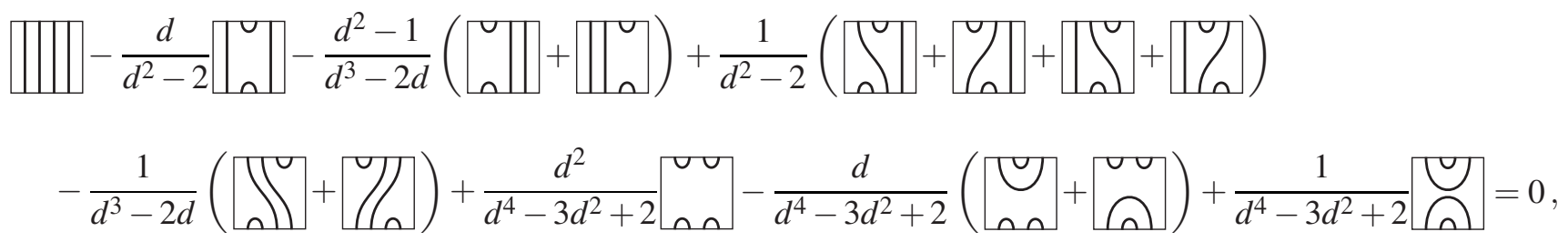

see Ref. 23 for a recursive formula. These relations define a finite-dimensional Hilbert space $V_{d}(Y) \subset \bar{V}_{d}(Y) \subset \mathrm{C}^{[S]} \subset \mathrm{C}^{S}$.

In Refs. 12 and 15 it is explained that $V_{d}(Y)$ is the Hilbert spaces for doubled $S U(2)_{k}$ Chern-Simons theory on $Y$. It has been argued $^{14}$ that a Hamiltonian with a ground-state manifold corresponding to $\bar{V}_{d}$ is perched at a phase transition. When perturbed, (infinitesimally for $k=1$ or 2 and under a larger deformation for $k \geq 3$ ) it will go into a topological phase with low-energy Hilbert space $V_{d}$, i.e., into a phase described by doubled $S U(2)_{k}$ Chern-Simons theory. Very briefly, the Hilbert space of a TQFT such as doubled $S U(2)_{k}$ Chern-Simons theory can always be defined as the joint null space of commuting local projectors ${ }^{24}$ implying the existence of a local Hamiltonian with a spectral gap in the thermodynamic limit. Once a Hamiltonian $H_{d}$ has imposed $d$-isotopy, i.e., $\operatorname{GSM}\left(H_{d}\right)=\bar{V}_{d}$, an extensive degeneracy has been created; The only local way of lifting this extensive degeneracy (to a finite degeneracy) without creating frustration ${ }^{25}$ is to add the Jones-Wenzl projector to such a Hamiltonian.

\section{MICROSCOPIC LATTICE MODELS}

We now briefly review some examples of microscopic Hamiltonians whose ground state(s) are described by $d$-isotopy. These Hamiltonians may not be particularly realistic (e.g., the two spin Hamiltonians presented here do not conserve the total spin) but they have the advantage of being relatively simple local Hamiltonians with the desired ground states. These ground states have a nice property that their square norms are in one-to-one correspondence to the partition functions of known statistical mechanical models-a socalled plasma analogy. ${ }^{26,27}$ This approach has proved useful in studying many frustrated quantum models and, in particular, those with topological and quasitopological orders. ${ }^{28-34}$

The first example was presented in Ref. 14 and was inspired by Kitaev's model. ${ }^{10}$ The model is defined on a honeycomb lattice with the elementary degrees of freedom being $s=1 / 2$ spins situated on its links (alternatively, one can think of these spins as occupying the sites of a kagomé lattice, but the former description lends itself nicer to a loop representation). The Hamiltonian is given by

$$
\begin{aligned}
H_{d}^{(1)}= & \sum_{v}\left[1+\prod_{i \in \mathcal{N}(v)} \sigma_{i}^{z}\right]+\sum_{p}\left[\frac{1}{d^{2}}\left(F_{p}^{0}\right)^{\dagger} F_{p}^{0}+F_{p}^{0}\left(F_{p}^{0}\right)^{\dagger}-\frac{1}{d} F_{p}^{0}\right. \\
& -\frac{1}{d}\left(F_{p}^{0}\right)^{\dagger}+\left(F_{p}^{1}\right)^{\dagger} F_{p}^{1}+F_{p}^{1}\left(F_{p}^{1}\right)^{\dagger}-F_{p}^{1}-\left(F_{p}^{1}\right)^{\dagger}+\left(F_{p}^{2}\right)^{\dagger} F_{p}^{2} \\
& +F_{p}^{2}\left(F_{p}^{2}\right)^{\dagger}-F_{p}^{2}-\left(F_{p}^{2}\right)^{\dagger}+\left(F_{p}^{3}\right)^{\dagger} F_{p}^{3}+F_{p}^{3}\left(F_{p}^{3}\right)^{\dagger}-F_{p}^{3}
\end{aligned}
$$

$$
\left.-\left(F_{p}^{3}\right)^{\dagger}\right],
$$

where $\mathcal{N}(v)$ is the set of three links neighboring vertex $v$, and

$$
\begin{gathered}
F_{p}^{0}=\sigma_{1}^{-} \sigma_{2}^{-} \sigma_{3}^{-} \sigma_{4}^{-} \sigma_{5}^{-} \sigma_{6}^{-} \\
F_{p}^{1}=\sigma_{1}^{+} \sigma_{2}^{-} \sigma_{3}^{-} \sigma_{4}^{-} \sigma_{5}^{-} \sigma_{6}^{-}+\text {cyclic perm. } \\
F_{p}^{2}=\sigma_{1}^{+} \sigma_{2}^{+} \sigma_{3}^{-} \sigma_{4}^{-} \sigma_{5}^{-} \sigma_{6}^{-}+\text {cyclic perm } . \\
F_{p}^{3}=\sigma_{1}^{+} \sigma_{2}^{+} \sigma_{3}^{+} \sigma_{4}^{-} \sigma_{5}^{-} \sigma_{6}^{-}+\text {cyclic perm. }
\end{gathered}
$$

Here, $1,2, \ldots, 6$ label the six edges of plaquette $p$. This Hamiltonian is a sum of projection operators with positive coefficients and therefore is positive definite. The eigenvalues of the first term in Eq. (5) are two and zero corresponding, respectively, to whether there is an even or odd number of $\sigma^{z}=-1$ spins neighboring this vertex. When eigenvalue zero is obtained at every vertex, the $\sigma^{z}=1$ links form loops. Hence, the zero-energy subspace of the first term is spanned by all configurations of multiloops (on the honeycomb lattice, they cannot cross).

The term on the second line of Eq. (5) is a projection operator which annihilates a state $|\Psi\rangle$ if the amplitude for all of the spins on a given plaquette to be up is a factor of $d$ times the amplitude for them all to be down, i.e., if the amplitude for a configuration with a small loop encircling a single plaquette is a factor of $d$ times the amplitude for an otherwise identical configuration without the small loop. The other three lines of the Hamiltonian vanish on a state $|\Psi\rangle$ if it accords the same value to a configuration if a loop is deformed to enclose an additional plaquette; in other words, these terms enforce the usual isotopy relations. These terms are graphically represented in Fig. 2.

The second example was presented in Ref. 15 motivated by the connection to a classical statistical mechanical model, namely, the self-dual Potts model (this connection will be explored in Sec. IV). The model is defined on a square lattice and the degrees of freedom are once again $s=1 / 2$ spins situated on its links. The Hamiltonian is given by 


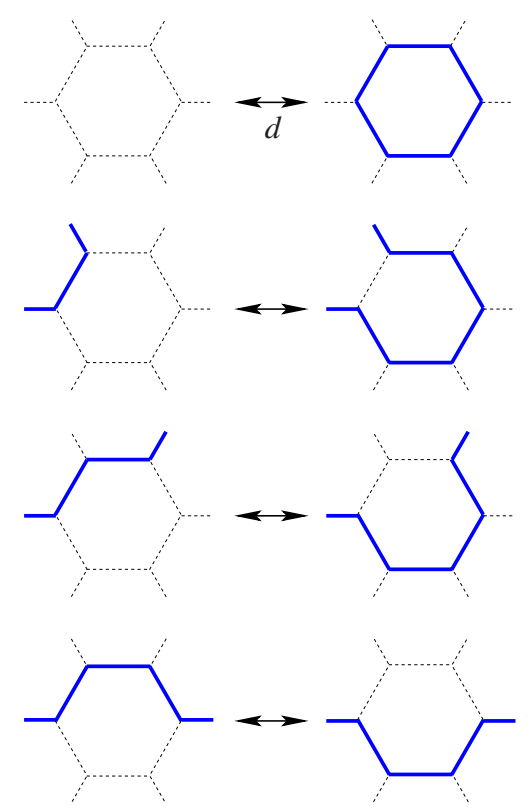

FIG. 2. (Color online) The action of the terms in the last four lines of the Hamiltonian (5) represented graphically. The solid bonds correspond to the up spins. Notice that the application of the Hamiltonian to any of the above plaquette configurations results in a superposition of this configuration and its counterpart with the appropriate amplitudes. The relative amplitudes of these configurations in the ground (zero eigenvalue) state correspond to the " $d$ "-isotopy rules described in Sec. I.

$$
\begin{aligned}
H_{d}^{(2)}= & \sum_{\square}\left(|3\rangle-\frac{1}{d}|4\rangle\right)\left(\langle 3|-\frac{1}{d}\langle 4|\right) \\
& +\sum_{+}\left(|\hat{1}\rangle-\frac{1}{d}|\hat{0}\rangle\right)\left(\langle\hat{1}|-\frac{1}{d}\langle\hat{0}|\right)
\end{aligned}
$$

in the notation of Ref. 15. The sum in Eq. (7) is taken over all elementary plaquettes of the lattice, $|3\rangle$ is a state with (any) three up spins around a given plaquette, and $|4\rangle$ has all four spins up. The second sum is taken over all vertices; $|\hat{1}\rangle$ and $|\hat{0}\rangle$ correspond to a single spin or no spins up around a given vertex. Notice that this Hamiltonian is self-dual under flipping all spins and going to the dual lattice.

The loops are now defined on the surrounding (or midpoint) lattice, i.e., the lattice obtained by connecting the midpoints of adjacent edges. One should think of placing a double-sided mirror along a bond whose spin is up and placing a mirror along a dual bond if the spin is down. Loops are formed by propagating light in this labyrinth of mirrors. The action of the first term [Eq. (7)] is demonstrated in Fig. 3. The action of the second (vertex) term is completely analogous due to the aforementioned duality.

Finally, a reader interested in a more realistic Hamiltonian is referred to Refs. 13 and 35 where a possibility of finding a $d$-isotopy ground state(s) in an extended Hubbard model is discussed. We shall refrain from reviewing that construction here due to its complexity which is unnecessary for the purpose of this paper.

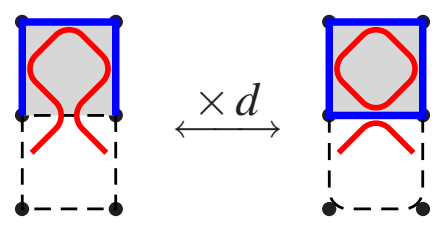

FIG. 3. (Color online) The action of the first (plaquette) term of Hamiltonian (7) on a shaded plaquette. A small loop is created or annihilated (with the appropriate amplitude) by being merged with another loop.

Let us turn to the common features of the above Hamiltonians. Both the terms on the final four lines of Eq. (5) and the plaquette and vertex terms of Eq. (7) do not commute with each other. However, they are compatible in the sense that each of these terms annihilates the following state:

$$
\left|\Psi_{0}\right\rangle=\sum_{\{\alpha\}} d^{\ell(\alpha)}|\alpha\rangle,
$$

which is a superposition of all configurations $\alpha$ of multiloops weighted by a factor of $d$ to the number of loops $\ell(\alpha)$.

Notice one important difference between these examples: By construction, in the second case the loops are fully packed; every edge of a surrounding lattice is traversed by a loop. Because of this constraint, the notion of simple isotopy is meaningless here; the multiloops, however, can fluctuate by "ejecting" and "absorbing" small loops. On an $L \times L$ torus, the ground-state degeneracy is $\sim L^{2}$ because the Hamiltonian does not mix states $|\alpha\rangle$ with different winding numbers. ${ }^{36}$ The different ground states are given by Eq. (8) but with the sum over $\alpha$ restricted to a single topological class. Notice that at least in the first example, the Hamiltonian dynamics is ergodic within a given topological sector; this is particularly trivial to see for the zero-winding case since every loop configuration can be reduced to a configuration with no loops by applying the moves depicted in Fig. 2 to first shrink the loops to a single plaquette and then to annihilate them.

In the second model, ergodicity is less obvious since the loop model is fully packed and thus reducing any given configuration to a state with no loops is impossible. Moreover, it has been observed in Ref. 15 that for a nonzero winding number for certain finite tori there are configurations that are not connected by the "moves" of the Hamiltonian (7). For the zero-winding number, however, it can be shown that all configurations are connected to the state with all spins down, which in turn translates into a maximum possible number of loops: one per each plaquette of a dual lattice. Hence the Hamiltonian is ergodic in the zero-winding sector and this is the sector we will be concerned with for the remainder of the paper. (Ergodicity is important because the twisted states we propose should have nothing to do with degeneracies associated with possible nonergodicity.)

More generally, the ground state on any genus $g \geq 1$ surface is infinitely degenerate in the thermodynamic limit. As we saw in Sec. II if $d= \pm 1$ or $d= \pm \sqrt{2}$, there is a JonesWenzl projector which also annihilates the ground state (8) on a topologically trivial manifold but mixes different winding number sectors on higher-genus surfaces. Hence, in ei- 
ther model at $d= \pm 1, \pm \sqrt{2}$, there are two Hamiltonians which have the same ground state on the sphere (or, equivalently, in the zero-winding sector). The first ones given by either Eqs. (5) or (7) have extensively degenerate ground states on the torus while the other kind (with the JonesWenzl projector added) have finite degeneracy. ${ }^{10}$ The second type leads to a topological phase with an energy gap for $d$ $=1$ (Ref. 10) where the resulting model is exactly soluble, and, we believe, for $d=-1$ (the situation at $d= \pm \sqrt{2}$ is more subtle and will be addressed later). The spectrum of the first kind is the main subject of this paper.

While the ground state can be obtained exactly, excited states cannot because the different operators in both Eqs. (5) and (7) do not commute with each other. We will use a variational ansatz to show that in the absence of other terms, such as Jones-Wenzl projectors, the spectra of these Hamiltonians are gapless in the zero-winding sector. But before we can proceed, we need to address the inherent structure of these ground states in more details.

\section{MAPPING OF THE GROUND STATE TO A STATISTICAL MECHANICS PROBLEM}

Many properties of the ground-state wave function can be obtained by observing that the norm of the ground state is equal to the partition function of a classical loop model,

$$
\left\langle\Psi_{0} \mid \Psi_{0}\right\rangle=\sum_{\{\alpha\}} d^{2 \ell(\alpha)},
$$

where $\alpha$ denotes a particular configuration of loops on a lattice (a "snapshot" of multiloops). The specific details of possible configurations depend on a particular choice of a Hamiltonian; in what follows we shall consider the cases relevant to each of the proposed Hamiltonians.

\section{A. Potts model: random clusters and loops}

The $q$-state Potts model originally introduced as a generalization of the Ising model is defined by the following classical Hamiltonian:

$$
-\beta \mathcal{H}=J \sum_{\langle i, j\rangle} \delta_{\sigma_{i}, \sigma_{j}}
$$

where the sum is taken over all pairs of nearest neighbors and $\sigma_{i}$ is a discrete "spin" variable that can take on $q$ different values, e.g., $\sigma_{i}=1, \ldots, q$.

We now review the Fortuin-Kasteleyn (FK) (also known as random cluster) representation for the $q$-state Potts model and underline its basic properties.

Given the Hamiltonian (10), the partition function can then be written as

$$
\begin{aligned}
Z_{\text {Potts }}=\sum_{\{\sigma\}} e^{-\beta \mathcal{H}} & =\sum_{\{\sigma\}} \prod_{\langle i, j\rangle} e^{J \delta_{\sigma_{i}, \sigma_{j}}} \\
& =\sum_{\{\sigma\}} \prod_{\langle i, j\rangle}\left[1+\left(e^{J}-1\right) \delta_{\sigma_{i}, \sigma_{j}}\right] \\
& =\sum_{\{\sigma\}} \prod_{\langle i, j\rangle}\left[1+v \delta_{\sigma_{i}, \sigma_{j}}\right],
\end{aligned}
$$

with $v \equiv e^{J}-1$
The next step is to expand the product in Eq. (11). Every pair of nearest neighbors contributes a factor of either 1 or $v$ to each of the resulting terms with the latter possibility available only if the neighboring spins agree $\left(\sigma_{i}=\sigma_{j}\right)$. Therefore every such term has a simple graphical representation: A bond is occupied if the pair of spins it connects contributes a factor of $v$ into a given term and it is left vacant otherwise. Since a bond can be placed between the sites only if their spins "agree," all spins belonging to the same bond cluster must have the same value.

The partition function thus becomes

$$
Z_{\mathrm{Potts}}=\sum_{\{\sigma\}} \prod_{\langle i, j\rangle}\left[1+v \delta_{\sigma_{i}, \sigma_{j}}\right]=\sum_{\{\sigma\}} \sum_{\{\omega\}} v^{b(\omega)} \Delta(\sigma, \omega),
$$

where $b(\omega)$ is the total number of occupied bonds in a bond configuration $\omega$, and $\Delta(\sigma, \omega)$ is the appropriate collection of Kronecker delta symbols that enforces the "agreement" between the spin and the bond configurations.

The next step is to change the order of summation in Eq. (12) (which is fine as long as the lattice is finite) and then sum over all spin configurations $\sigma$. The only constraint on the spin variables (for a fixed bond configuration $\omega$ ) is the one that has been mentioned earlier: all connected spins must take on the same value (one of the $q$ possible). Therefore every connected cluster, as well as each isolated site, contributes a factor of $q$ to the resulting bond weight, and the partition function becomes

$$
Z_{\text {Potts }}=\sum_{\{\omega\}} v^{b(\omega)} q^{c(\omega)},
$$

where $c(\omega)$ is the total number of connected components (including isolated sites). The partition function (13) defines FK (random-cluster) representation. ${ }^{37,38}$

While all previous considerations applied to any number of spatial dimensions, in 2D the partition function (13) can also be written in a way which makes its self-dual property apparent. In order to do this, notice that if we think of an unoccupied bond of the actual lattice as an occupied bond of the dual lattice (therefore $b^{*}=B-b$ ) then every circuit (face) of the actual lattice contains a dual connected component: $f=c^{*}$ [here we define $b^{*} \equiv b\left(\omega^{*}\right)$ and $c^{*} \equiv c\left(\omega^{*}\right)$ with $\omega^{*}$ denoting the configuration of dual bonds]. Therefore, using the Euler relation (for a planar graph)

$$
f(\omega)=b(\omega)+c(\omega)-N,
$$

with $f(\omega)$ being the number of circuits (defined as a minimum number of bonds that one has to cut in order to make a graph consist only of trees $^{39}$ ) and $N$ being the total number of sites, we can rewrite Eq. (13) as

$$
Z_{\text {Potts }}=\sum_{\{\omega\}} v^{f-c+N} q^{c}=v^{N} \sum_{\{\omega\}} v^{f}\left(\frac{q}{v}\right)^{c}=v^{N} \sum_{\{\omega\}} v^{c^{*}}\left(\frac{q}{v}\right)^{c} .
$$

If we ignore the uninteresting analytic prefactor in Eq. (15), we immediately see that the system is self-dual when $v$ $=q / v$, i.e., $v=\sqrt{q}$.

A so-called polygon decomposition ${ }^{40}$ lets us relate random clusters to a loop gas on the surrounding lattice. We 


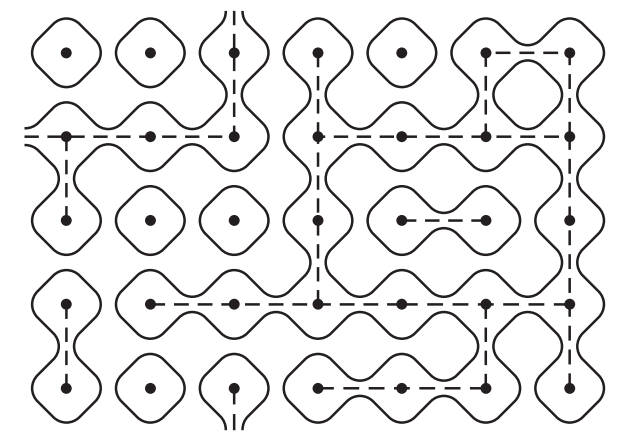

FIG. 4. A typical cluster configuration for the Potts model is shown by dashed lines. Spins belonging to the same cluster take the same value, which must be summed over the $q$ possible values, as described in the text. Clusters can be represented by loops on the surrounding lattice shown by solid lines.

think of an occupied bond as a double-sided mirror placed at the site of the surrounding lattice. If a bond is not occupied, then its dual bond is considered a mirror. Thus every site of the surrounding lattice gets one of the two possible mirrors. We then use these mirrors to construct paths as shown in Fig. 4. Since these paths have no sources or sinks, they always form loops that either surround the clusters or are contained inside clusters (in the latter case, the loops surround dual clusters). The number of loops $\ell$ is then given by $\ell=c+c^{*}$. If $v=\sqrt{q}$-i.e., if the Potts model is at its self-dual point, then

$$
Z_{\text {self-dual }}=\sum_{\{\alpha\}}(\sqrt{q})^{\ell(\alpha)},
$$

where the sum is taken over all fully packed loop configurations on the surrounding lattice. Clearly, with the choice of $q=d^{4}$, the partition function (16) becomes the norm of the ground state $\left\langle\Psi_{0} \mid \Psi_{0}\right\rangle$ of the quantum Hamiltonian $H_{d}^{(2)}$.

\section{B. Random clusters on a torus}

In this section we will take the reader through some mathematical details in order to establish certain properties of the critical FK model. These properties will be relied upon later in the course of demonstrating the central result of this paper.

For the sake of concreteness, let us focus the FK representation of the critical $q$-state Potts model $(1 \leq q \leq 4)$ on a square lattice with periodic boundary conditions in both directions, i.e., the torus. In the case of $q=1$, the statistical mechanical model reduces to critical bond percolation. In this section we are focusing on the FK clusters established in Sec. IV A.

Let us begin by proving:

Proposition 1. Fixing $1 \leq q \leq 4$ and $\lambda>0$ there is an $\varepsilon>0$ so that for all $L$ sufficiently large there is a probability greater than $\varepsilon$ that the largest $\mathrm{FK}_{q}$ cluster in the $L \times L$ torus has Euclidian diameter $<\lambda L$.

Proof. The technology for this type of result was discovered and developed by the 1990s in the context of critical percolation ${ }^{41-44}$ but it can be extended to other critical systems provided analogs of the Russo-Seymour-Welsh (RSW) inequality on crossing probabilities for rectangles and of the Fortuin-Kasteleyn-Ginibre (FKG) inequality ("monotone

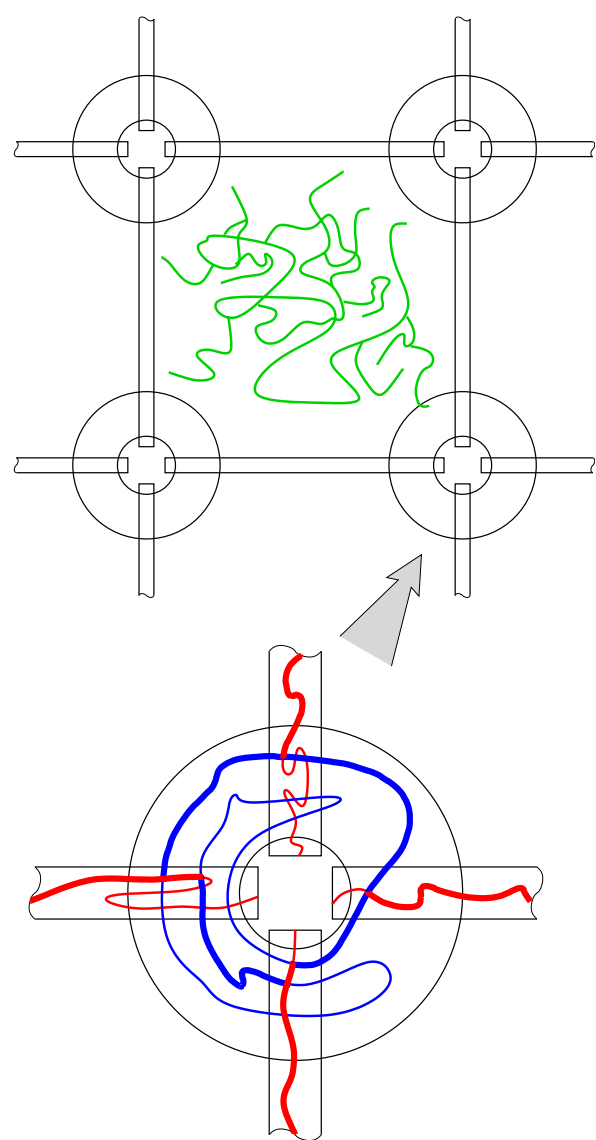

FIG. 5. (Color online) Construction of a wiggly approximation $G_{w}$ to a rectangular grid as described in the text. $G_{w}$ is built as a subset of the dual clusters; the goal of the construction is to "trap" the actual FK clusters inside $G_{w}$ as shown here.

events are positively correlated") hold. The proof strategy is to build some wiggly approximation $G_{w}$ lying entirely in the complement of FK clusters, to a rectilinear grid of scale (roughly) $\frac{L}{2 \lambda} \cdot G_{w}$ must not be too wiggly as we need the boxes in its complement to have diameter $<\lambda$ (see Fig. 5). Since each FK cluster lies in some such box, the clusters also have diameter $<\lambda$.

Building the grid $G_{w}$ is a random construction and must succeed with probability $>\varepsilon$. The naive idea for building the grid is to first condition on no clusters meeting the (roughly) $\left(\frac{L}{2 \lambda}\right)^{2}$ sites (i.e., four-coordinated vertices) of the desired grid $G_{S}$. Then, use RSW to further condition on cluster-disjoint arcs lying in small rectangles near the (roughly) $2\left(\frac{L}{2 \lambda}\right)^{2}$ bonds of $G_{S}$. What goes wrong is that the individual sites are simply too small; in the analogous electromagnetic problem the capacitance vanishes in the thermodynamic limit. The resolution is to consider small annuli enclosing each site and substitute for the site condition the event that an essential circle percolates around the annulus (also an RSW result).

Now, elementary planar topology allows bits and pieces of the annular and rectangular percolation paths to be hooked together to build the desired $G_{w}$ as shown in Fig. 5. Throughout this construction, it is essential that these percolation events are at least non-negatively correlated (we remind the readers that the properties of dual clusters are identical to 
those of the actual FK clusters at criticality). This is precisely what the FKG inequality provides. ${ }^{41,42}$

An immediate consequence of proposition 1 is that there is a nonzero probability that an arbitrary multiloop is in the zero-winding-number sector. Thus, we may confine our discussion to that sector and use the measure on that sector induced by the measure $d^{2 \ell(\alpha)}$ on all multiloops.

Let us pause for some definitions. Given a loop $a$ in the torus $T$, let $\|a\|_{x}$ or simply $\|a\|$ for short be the "breadth in the $x$ direction of $\widetilde{a}$ ' where $\widetilde{a}$ represents a (any) lift of $a$ up to the universal cover $\mathbb{R}^{2} \rightarrow T^{2}$. Recall that the universal cover of the torus $T^{2}$ is constructed by unwrapping completely in both the $x$ and $y$ directions. Very concretely, we may build the cover $\mathbb{R}^{2}$ from our original $L \times L$ square by taking a copy for each Gaussian integer and gluing these together (corresponding to Gaussian integers differing by one or $i$ ) to form a tiling of the plane. (Technically, it is the discrete plane $\mathbb{Z}^{2}$ that we produce.) A loop $a$ in $T^{2}$ may be regarded as a map from the circle $S^{1}$ into $T^{2}$, and a lift $\tilde{a}$ is any map to $\mathbb{R}^{2}$ making the following diagram commute:

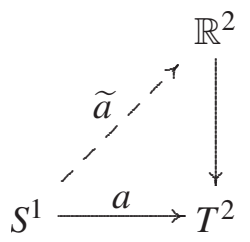

Concretely, the image of $\tilde{a}$ is obtained by taking an arc in the $L \times L$ square and following its continuation in the tiling of $\mathbb{R}^{2}$ until it closes back on itself. The assumption that the winding sector is trivial means any such $\tilde{a}$ is finite and in fact contains exactly as many bonds as $a$ contained in $T^{2}$, i.e., $<2 L^{2}$.

To return to the definition of $\|a\|$, we say $\|a\|=\max \mid x_{i}$ $-x_{j} \mid$, where the maximum is taken over all sites $\left(x_{i}, y_{i}\right)$ on a lift $\tilde{a}$. Note that any two lifts $\tilde{a}$ and $\tilde{a}$ are congruent by a translation of $\mathrm{R}^{2}$ so $\|a\|$ is well defined.

Finally, for a multiloop $\alpha \subset T^{2}$ we define $\|\alpha\|$ $=\max _{a \subset \alpha}\|a\|$. We are now ready to state a proposition about the diameter of these unwrapped components of random multiloops from the trivial sector.

Proposition 2. In the scaling limit, $\|\alpha\|$ is almost surely a bounded function on the trivial winding sector. More precisely, the probability that $\|\alpha\|>r L$ has an upper bound which is independent of $L$.

Proof. We need a geometric lemma.

Lemma 1. Let $T$ be a Euclidian torus of area 1. Let $\gamma:[0,1] \rightarrow T$ be an arc so that the (Euclidian) distance between lifted end points in the universal cover $d[\widetilde{\gamma}(0), \widetilde{\gamma}(1)]$ $=\delta$. Then there is a nontrivial deck translation (additive action of a Gaussian integer) $\widetilde{\gamma}^{\prime}$ of $\tilde{\gamma}$ so that $\operatorname{dist}\left(\tilde{\gamma}, \widetilde{\gamma}^{\prime}\right)<\frac{1}{\delta}$.

Proof. Let $X$ be the radius $r$ neighborhood of $\gamma$ in the cover $\mathbb{R}^{2}$. Integrating the lengths of slices of $X$ perpendicular to the straight-line segment $[\widetilde{\gamma}(0), \widetilde{\gamma}(1)]$, we see (by Fubini's theorem) that area $(X)>2 r \delta$ (where area is counted without multiplicity). If $X$ is disjoint from its deck transformations then it descends one-to-one into the torus implying $\operatorname{area}(X)$ $<1$. Thus, $2 r \delta<1$ and so $r<\frac{1}{2 \delta}$.

Now apply the lemma to arcs $\gamma$ within an FK cluster $K$ (in the trivial sector) on $T$. Since we are in the trivial sector, we may define $\|K\|$ exactly as we defined the norm on loops. By the lemma, if $\|K\|=\delta, K$ will come within distance $1 / \delta$ of completing a nontrivial wrapping of the torus. An application of the RSW inequality shows that it is unlikely (vanishing algebraically in $1 / \delta$ ) to come close to wrapping $T^{2}$ but fail to wrap completely. RSW amounts to integrating the effect of bringing bonds, which may join large clusters, in and out of our FK snapshot. Such fluctuations cannot be created by any local $H_{d}$ for $q \neq 1$ since the weight of the update is nonlocal. Nevertheless, these fluctuations do preserve the measure $d^{2 \ell(\alpha)}$. So, the fraction of critical, topologically trivial, and $\mathrm{FK}_{q}$ snapshots $(1 \leq q \leq 4)$ which contain a cluster $K$ with $\|K\|>\delta$ is $\frac{1}{\operatorname{poly}(\delta)}$.

\section{C. $\mathbf{O}(n)$ loop model}

Another model describing the statistics of loops on a lattice is the so-called $\mathrm{O}(n)$ loop model. ${ }^{45}$ Let us begin by defining the following $\mathrm{O}(n)$ spin model on some (finite) lattice by the following partition function:

$$
Z_{\mathrm{O}(n)}(x)=\int \prod_{i} \frac{d \hat{S}_{i}}{\Omega_{n}} \prod_{\langle i, j\rangle}\left(1+x \mathbf{S}_{i} \cdot \mathbf{S}_{i j}\right),
$$

with $\mathbf{S}_{i} \in \mathbb{R}^{n},\left|\mathbf{S}_{i}\right|=1$, and $\Omega_{n}$ is the $n$-dimensional solid angle. In order to obtain the loop model, we write $\mathbf{S}_{i} \cdot \mathbf{S}_{j}=S_{i}^{(1)} S_{j}^{(1)}$ $+\ldots+S_{i}^{(n)} S_{j}^{(n)}$ and define $n$ as different colors (each of which will be associated with a specific component of the $\mathrm{O}(n)$ spins). Multiplying out all terms in Eq. (17), we have $n$ choices for each bond plus a possibility of a vacant bond. Thus, the various terms are represented by an $n$-colored bond configuration: $\mathcal{G}=\mathcal{G}_{1}, \ldots \mathcal{G}_{n}$ with $\mathcal{G}_{\ell}$ denoting those bonds where the term $S_{i}^{(\ell)} S_{j}^{(\ell)}$ has been selected. Clearly, the various $\mathcal{G}_{\ell}$ 's are pairwise (bond) disjoint. Thus, for each $\mathcal{G}$ we obtain the weight

$$
W_{\mathcal{G}}=\operatorname{Tr} \prod_{\langle i, j\rangle \in \mathcal{G}_{1}} x S_{i}^{(1)} S_{j}^{(1)} \cdots \prod_{\langle i, j\rangle \in \mathcal{G}_{n}} x S_{i}^{(n)} S_{j}^{(n)} .
$$

On the basis of elementary symmetry considerations it is clear that $W_{\mathcal{G}} \neq 0$ if and only if each vertex houses an even number (which could be zero) of bonds of each color. Once this constraint is satisfied, we get an overall factor of $x^{b(\mathcal{G})}$ — with $b(\mathcal{G})$ being the total number of bonds times the product of the vertex factors obtained by performing the appropriate $\mathrm{O}(n)$ integrals. Obviously, these vertex factors depend only on how many different colors and how many of each of these colors enter each vertex (i.e., not on the particular colors involved nor on the directions of approach to the vertex). A particularly easy case is that of the honeycomb lattice where, due to a low coordination number, a maximum of two bonds of a single color can visit a vertex. The corresponding vertex factor is then given by

$$
\int \frac{d \hat{S}_{i}}{\Omega_{n}}\left(S_{i}^{(j)}\right)^{2}=\frac{1}{n}
$$

leading (after summing over all $n$ colors) to the following expression for the partition function: 


$$
Z_{\mathrm{O}(n)}(x)=\sum_{\{\alpha\}}\left(\frac{x}{n}\right)^{b(\alpha)} n^{\ell(\alpha)}
$$

where $b(\alpha)$ is the total number of occupied bonds (the total perimeter of all loops) while $\ell(\alpha)$ is the total number of loops. The last factor appears because each loop could be of one of $n$ colors. The expression in Eq. (20) is well defined for arbitrary $n$ and $x$ so it can be taken as the definition of the $\mathrm{O}(n)$ loop model. ${ }^{46}$

We note that the partition function (20) is exactly the norm of the ground state $\left\langle\Psi_{0} \mid \Psi_{0}\right\rangle$ of the quantum Hamiltonian $H_{d}^{(1)}$ given by Eq. (5) provided that $x=n$ and $d^{2}=n$.

\section{Correlations}

Both of these models have a Coulomb gas representation ${ }^{46}$ so that their correlation functions can be obtained from exponential operators in a Gaussian field theory with a background charge. Consider, for instance, the $\mathrm{O}(n)$ model. Precisely the same loop expansion derived in Sec. IV C can be obtained from an silicon-on-sapphire (SOS) model on the kagomé lattice. After integrating out the triangular faces, the resulting SOS model for the heights on the hexagonal faces has a low-temperature expansion which is a sum over domain-wall configurations. The weights of the SOS model (or, equivalently, 6-vertex model) are such that when a domain wall turns left, it acquires a factor $(x / n) e^{i \chi}$; when it turns right, a factor $(x / n) e^{-i \chi}$. Since a loop will only close if the difference between the number of right terms and the number of left turns is \pm 6 , every closed loop receives a factor $(x / n)^{b} e^{ \pm 6 i x}$ where $b$ is the length of the loop. Summing over both orientations of the loop, we obtain $2(x / n)^{b} \cos 6 \chi$. Hence, this is equivalent to the $\mathrm{O}(n)$ loop model for $n<2$ if we take $n=2 \cos 6 \chi$. When $x>x_{c}$ $=n / \sqrt{2+\sqrt{2-n}}$, this model is in its low-temperature phase, which is critical.

The SOS model is a Coulomb gas with coupling $g=1$ $-6 \chi / \pi$ and background charge $-2(1-g)$ (which ensures the correct phase factor for each turn of a loop). Consider the $\left\langle\mathbf{S}_{i} \cdot \mathbf{S}_{j}\right\rangle$ correlation function in the $\mathrm{O}(n)$ model. Every configuration which gives a nonzero contribution must have one curve which does not close into a loop but has end points at spins $i$ and $j$. In Coulomb gas language, they have magnetic charges $\pm 1 / 2$. In addition, they must each also have electric charge $1-g$. Together with their magnetic charge, this will ensure that a factor $e^{ \pm 6 i \chi}$ arises whenever the curve winds around either point (as required by the SOS vertex rules). This electric charge also cancels the background charge. Thus, the Coulomb gas operators corresponding to $\mathrm{O}(n)$ spin operators have electric and magnetic charges $(1-g, \pm 1 / 2)$. The exponent associated with a correlation function between field with electric and magnetic charges $\left(e_{1}, m_{1}\right)$ and $\left(e_{2}, m_{2}\right)$ is $x_{e_{1}, m_{1} ; e_{2}, m_{2}}=-e_{1} e_{2} / 2 g-g m_{1} m_{2} / 2$. Hence, the $\mathrm{O}(n)$ spinspin correlation function has the power-law decay, ${ }^{46}$

$$
\langle\mathbf{S}(r) \cdot \mathbf{S}(0)\rangle \sim \frac{1}{r^{x_{M}}},
$$

where $x_{M}=\frac{1}{4} g-\frac{1}{g}(1-g)^{2}$.
The loops which surround random clusters as described in Sec. IV A in the self-dual critical $q$-state Potts model can also be mapped onto a 6-vertex model (and, therefore, a Coulomb gas) in a similar fashion. Precisely the same exponents are obtained.

Although, they are crucial to our proof of gaplessness, these correlation functions are not the ones of direct physical interest. Physical correlation functions have a rather different behavior. Consider equal-time correlations between quantum spins such as $\left\langle\sigma_{i}^{z} \sigma_{j}^{z}\right\rangle$ in the original quantum models (5) and (7). As we now argue, they are short-ranged in space. In the first model, the equal-time $\left\langle\sigma_{i}^{z} \sigma_{j}^{z}\right\rangle$ correlation function is related to the probability that a loop passes through $i$, and a loop which may or may not be distinct passes through $j$. Such correlation functions vanish in the $\mathrm{O}(n)$ loop models. Analogously, in the second case, this correlation function is related to the probability that the two corresponding bonds are parts of clusters but not necessarily in the same cluster in the related $q$-state Potts model. Such a correlation function once again vanishes.

In Coulomb gas language for the associated statistical mechanical models, the reason that such correlation functions vanish is that they are correlation functions of electrically neutral operators such as gradients of the height (to which the local loop density corresponds). Such correlation functions vanish since they do not cancel the background charge. (The only exception is a height model with central charge $c=1$ for which there is no background charge. Gradients of the height have power-law correlations.) As we have seen, algebraic decay is possible for correlation functions of operators which are charged in the Coulomb gas picture; but these are nonlocal in terms of the spins $\sigma_{i}^{z}$ since they measure, for instance, the probability that two spins $\sigma_{i}^{z}$ and $\sigma_{j}^{z}$ lie on the same loop. (At $d=1, \sqrt{2}$, this can also be seen from the fact that the ground state on the sphere is the same-and therefore has the same equal-time correlation functions-as that of a gapped Hamiltonian ${ }^{10,47}$ which is a sum of local commuting operators and therefore has correlation length zero.)

Thus, the ground-state wave function of Eq. (5) has an underlying power-law long-ranged structure which is apparent in its loop representation, but it is not manifested in the correlation functions of local operators $\sigma_{i}^{z}$. As we will see momentarily, this long-range structure leads to gapless excitations for the Hamiltonian (5) and, therefore, long-ranged correlations in time in spite of the lack of long-ranged correlations in space. We call such a state of matter a quasitopological critical point.

\section{LOW-ENERGY EXCITATIONS}

In spite of the short-ranged nature of equal-time spin-spin correlation functions and the absence of any conservation laws for either of the Hamiltonians (5) and (7), we can construct a variational argument that this general type of Hamiltonians is gapless using the criticality of nonlocal correlation functions, specifically, the scale-invariant nature of loops.

The general idea of our proof is to produce a "twisted" state which is both orthogonal to the ground state and has a 
vanishingly small expectation value of the Hamiltonian in the general spirit of the Lieb-Schultz-Mattis theorem for quantum antiferromagnets. ${ }^{21,48,49}$

For the purposes of this theorem we do not need to choose a particular Hamiltonian but only require that it satisfies the following necessary conditions:

(i) This is a $d$-isotopy Hamiltonian whose ground state is described by Eq. (8) with a scale-invariant distribution of loops in the thermodynamic limit. We make the mathematically nontrivial assumption widely accepted in physics that the scaling limit exists. Furthermore, certain probability functions defined from the limit will be formally differentiated. These derivatives can easily be replaced by finite difference quotients so continuity is, in fact, an adequate assumption. (We shall make this condition more precise later.)

(ii) A second important feature of the ground state which we use is that when viewed as a statistical mechanical ensemble by $\operatorname{prob}\left(\psi_{i}\right)=\left|\psi_{i}\right|^{2}$, it is in the scaling limit a critical system with the "no large cluster property," i.e., on an $L$ $\times L$ square with periodic boundary conditions ("torus") $\forall \lambda$ $>0, \exists \varepsilon>0$ so that the event "all loops within the random multiloop $\psi_{i}$ have breadth (as defined in Sec. IV B) $<\lambda L$ " occurs with probability greater than $\varepsilon$. (We have seen that the FK models for $q \geq 1$ have this property. We also expect this property to hold for the $\mathrm{O}(n)$ loop models $1 \leq n \leq 2$ : proving this would be quite interesting.)

(iii) The Hamiltonian is local: all allowed $d$-isotopy moves have finite range (generalizing our theorem to the case of a quasilocal Hamiltonian whose terms decay exponentially with increasing range is straightforward). Without loss of generality, we might as well assume that the range of all terms is limited to a single lattice plaquette.

(iv) The terms responsible for the loop dynamics are bounded uniformly in the size of the system, i.e., for any two multiloops $\alpha$ and $\beta,\left|\left\langle\alpha\left|H_{d}\right| \beta\right\rangle\right|<V$. (We assume that the basis vectors $|\alpha\rangle$ of the Hilbert space of $H_{d}$ are orthonormal, i.e., $\langle\alpha \mid \beta\rangle=0$ unless $\alpha$ and $\beta$ are identical multiloops and $\langle\alpha \mid \alpha\rangle=1$.) Notice that we make no assumptions about the other terms that might be there to enforce the multiloop constraint - these can potentially include hard-core interactions.

There is little doubt that both of the presented Hamiltonians satisfy the above conditions. However, our argument is more complete in the second case $\left(\mathrm{FK}_{q}, 1 \leq q \leq 4\right)$.

We will now construct our low-energy excitation $\left|\psi_{1}\right\rangle$ above the ground state $\left|\psi_{0}\right\rangle$. We normalize so that $\left\langle\psi_{0} \mid \psi_{0}\right\rangle$ $=1,\left\langle\psi_{0}|H| \psi_{0}\right\rangle=0$, and $\left\langle\psi_{1} \mid \psi_{1}\right\rangle=1$. We will construct $\left|\psi_{1}\right\rangle$ and then a family of "harmonics" $\left|\psi_{k}\right\rangle$, all of norm one, and estimate the energy expectation values $\left\langle\psi_{k}|H| \psi_{k}\right\rangle, k \geq 1$. In constructing $\left|\psi_{k}\right\rangle$, we will use the language of the scaling limit for conceptual simplicity. It is quite routine and we leave this to the reader to back away from the scaling limit and write discrete formulae replacing derivatives with difference quotients.

We use $\mathcal{C}$ to denote a configuration (i.e., a multiloop) near the scaling limit, i.e., $L \gg 0$, on $T$ : the $L \times L$ torus. By proposition 2 we know there is a function $r_{\mathcal{C}}$ on configurations which remains almost surely defined in the scaling limit: $r_{\mathcal{C}}$ $\equiv\|\mathcal{C}\| / L$. Our assumption is that in the scaling limit, the probability $p$ that a configuration $\mathcal{C}$ satisfied $r_{\mathcal{C}} \leq r$ is a con- tinuous function $p(r)$. The probability is computed with respect to the $\mathrm{FK}_{q}$ measure, i.e., with respect to $\left|\psi_{0}\right\rangle$. As explained earlier, we will formally treat $p$ as differentiable writing $\frac{d p}{d r}$, but this may be treated as a difference quotient.

The ground-state wave function (on the trivial sector) $\left|\psi_{0}\right\rangle$ is simply

$$
\left|\psi_{0}\right\rangle=Z^{-1 / 2} \sum_{\alpha} d^{\ell(\alpha)}|\alpha\rangle
$$

where the sum is over all multiloops $\alpha$ in the zero-windingnumber sector and the normalization $Z=\Sigma_{\{\alpha\}} d^{2 \ell(\alpha)}$ is the partition function of the associated statistical mechanical model [Potts or $\mathrm{O}(n)]$.

We write the variational ansatz

$$
\left|\psi_{k}\right\rangle=Z^{-1 / 2} \sum_{\alpha} e^{2 \pi i k p\left(r_{\alpha}\right)} d^{\ell(\alpha)}|\alpha\rangle
$$

where $k$ is an integer. These states are orthonormal because

$$
\left\langle\psi_{k} \mid \psi_{l}\right\rangle=Z^{-1} \sum_{\alpha} d^{2 \ell(\alpha)} e^{2 \pi i p\left(r_{\alpha}\right)(k-l)}=\int_{0}^{1} d p e^{2 \pi i p(k-l)}=\delta_{k l} .
$$

In particular, $\left|\psi_{k}\right\rangle$ with $k \neq 0$ is orthogonal to the ground state. Hence, the expectation value of the Hamiltonian in this state is an upper bound on the energy gap between the ground and the first excited states

$$
\begin{aligned}
\Delta & \leq\left\langle\psi_{k}\left|H_{d}\right| \psi_{k}\right\rangle-\left\langle\psi_{0}\left|H_{d}\right| \psi_{0}\right\rangle \\
& =\frac{1}{Z} \sum_{\alpha, \beta}\left\{e^{2 \pi i k\left[p\left(r_{\alpha}\right)-p\left(r_{\beta}\right)\right]}-1\right\} d^{2 \ell(\alpha)}\left\langle\alpha\left|H_{d}\right| \beta\right\rangle .
\end{aligned}
$$

In the second line, we have used the fact that $\left\langle\alpha\left|H_{d}\right| \beta\right\rangle$ is nonzero only if $\ell(\alpha)=\ell(\beta)$. Exchanging $\alpha$ with $\beta$ exchanges each term in the sum in Eq. (25) with its complex conjugate. Hence, we can write

$$
\Delta \leq 2 \operatorname{Re}\left\{\frac{1}{Z} \sum_{\alpha, \beta ; r_{\beta}>r_{\alpha}}\left\{e^{2 \pi i k\left[p\left(r_{\alpha}\right)-p\left(r_{\beta}\right)\right]}-1\right\} d^{2 \ell(\alpha)}\left\langle\alpha\left|H_{d}\right| \beta\right\rangle\right\} .
$$

Note that we have dropped the case $r_{\beta}=r_{\alpha}$ since this expression vanishes for these configurations. The $d$-isotopy Hamiltonian can change the length of a loop by at most one lattice spacing, so $\left\langle\alpha\left|H_{d}\right| \beta\right\rangle \neq 0$ only if $r_{\beta}=r_{\alpha}+\frac{a}{L}$ where $a$ is the lattice spacing. (Recall that $r_{\alpha}$ has been defined in units of L.) Then, writing $p\left(r_{\alpha}\right)-p\left(r_{\beta}\right) \approx p^{\prime}\left(r_{\alpha}\right) \frac{a}{L}$, we have

$$
\Delta \leq 2 \operatorname{Re}\left\{\frac{1}{Z} \sum_{\alpha, \beta ; r_{\beta}>r_{\alpha}}\left(e^{2 \pi i k p^{\prime}\left(r_{\alpha}\right) a / L}-1\right) d^{2 \ell(\alpha)}\left\langle\alpha\left|H_{d}\right| \beta\right\rangle\right\} .
$$

Inserting $\int_{0}^{1} d r \delta\left(r-r_{\alpha}\right)=1$ into this expression, we have 


$$
\begin{aligned}
\Delta \leq & 2 \operatorname{Re}\left\{\int_{0}^{1} d r \delta\left(r-r_{\alpha}\right) \frac{1}{Z_{\alpha, \beta ; r_{\beta}>r_{\alpha}}}\left[e^{2 \pi i k p^{\prime}\left(r_{\alpha}\right) a / L}-1\right]\right. \\
& \left.\times d^{2 \ell(\alpha)}\left\langle\alpha\left|H_{d}\right| \beta\right\rangle\right\} \\
= & 2 \operatorname{Re}\left\{\int_{0}^{1} d r\left[1-e^{2 \pi i k p^{\prime}(r) a / L}\right] \bar{\rho}(r)\right\},
\end{aligned}
$$

where

$$
\begin{aligned}
\bar{\rho}(r) & \equiv-\frac{1}{Z} \sum_{\alpha, \beta ; r_{\beta}>r_{\alpha}} \delta\left(r-r_{\alpha}\right) d^{2 \ell(\alpha)}\left\langle\alpha\left|H_{d}\right| \beta\right\rangle \\
& \equiv \frac{1}{Z} \sum_{\alpha} \delta\left(r-r_{\alpha}\right) d^{2 \ell(\alpha)} \rho_{\alpha} .
\end{aligned}
$$

In the first line of Eq. (27), we define $\bar{\rho}(r)$, which is the expectation value of $\rho_{\alpha}$, defined in the second line. If we can argue that $\bar{\rho}(r) \leq A$ for some constant $A$ independent of $r$, then

$$
\begin{aligned}
\Delta & \leq 2 A \operatorname{Re}\left\{\int_{0}^{1} d r\left[1-e^{2 \pi i k p^{\prime}(r) a / L}\right]\right\} \\
& =2 A \int d r\left\{1-\cos \left[2 \pi i k p^{\prime}(r) a / L\right]\right\} \\
& \leq 2 A[1-\cos (2 \pi i k M a / L)] .
\end{aligned}
$$

Here, we have taken $p^{\prime}(r) \leq M$, which follows from our mild continuity assumptions. Consequently, in the large $L$ limit $\Delta \sim k^{2} / L^{2}$. Note that the $L^{-2}$ reproduces the classical scaling for the lowest eigenvalue of a string of length $L$, while the $k^{2}$ dependence signals a quadratic dispersion relation associated to a soft mode.

We are nearly finished. All that remains is to argue that $\bar{\rho}(r)$ defined by Eq. (27) satisfies $\bar{\rho}(r) \leq A$ for some constant $A$ independent of $r . \rho_{\alpha}$ is the number of distinct sites (i.e., distinct terms in $H_{d}$ ) where $H_{d}$ can produce a fluctuation stretching out the ( $x$ direction of) widest loop in the multiloop $\alpha$ from breadth $r_{\alpha}$ to breadth $r_{\alpha}+\frac{a}{L}$, i.e., by one lattice step. $\bar{\rho}(r)$ is the expectation value of $\rho_{\alpha}$ averaged over all configurations $\alpha$ whose widest loop has breadth $r$. Note that in the scaling limit $\bar{\rho}(r)$ can have no $r$ dependence. Prior to reaching the scaling limit, we use argument $r$ in $\rho(r)$ to indicate maximum cluster breadth in units of $\frac{1}{L}$. We now define $\rho_{\alpha, n}$ to be one if the widest loop $K \in \alpha$ meets the right side of the unique smallest rectilinear box $B$ containing $K$ and $K \subset B$ in $n$ distinct "fingers" touching the right-hand wall and zero otherwise. We define $\overline{\rho_{n}}(r)$ to be its expectation value,

$$
\overline{\rho_{n}}(r) \equiv \frac{1}{Z} \sum_{\alpha} \delta\left(r-r_{\alpha}\right) d^{2 \ell(\alpha)} \rho_{\alpha, n},
$$

then

$$
\bar{\rho}(r)=\sum_{n=1}^{\infty} n \overline{\rho_{n}}(r) .
$$

The Hamiltonian $H_{d}$ can produce $n$ distinct states contributing to $\bar{\rho}_{1}\left(r+\frac{a}{L}\right)$ for each state contributing to $\bar{\rho}_{n}(r)$. For example, in the figure above, one may fluctuate any rightmost finger further to the right. Hence,

$$
\bar{\rho}_{1}\left(r+\frac{a}{L}\right) \geq \sum_{n=1}^{\infty} n \rho_{n}(r) .
$$

But the right-hand side is simply $\bar{\rho}(r)$. Since $\bar{\rho}_{1}(r)$ is the ensemble average of $\rho_{\alpha, 1}=0,1$, it must satisfy $0 \leq \bar{\rho}_{1}(r) \leq 1$. Hence, if there is a well-defined scaling limit $\bar{\rho}_{1}(r) \rightarrow \rho_{1}, \bar{\rho}(r)$ and $\bar{\rho}\left(r+\frac{a}{L}\right)$ should converge. Then the latter is the desired constant $A$ in the upper bound (28) on the excited-state energy since we can rewrite Eq. (31) as

$$
\bar{\rho} \leq \bar{\rho}_{1} .
$$

Naturally, this is only an upper bound, and in fact we have not proved these are not just other degenerate ground states. However, it seems unlikely that we have discovered groundstate degeneracy (on the sphere) since there is no continuous symmetry of the Hamiltonian which could be broken or any other nonergodicity. Furthermore, we expect $\omega \propto k^{2}$ to be the correct behavior of the low-lying excitations; our intuition is based on observations made for other quasitopological models $s^{31,32,50}$ closely related to our $d=1$ case. Due to this characteristic quadratic spectrum, such lines of critical points have been dubbed "quantum Lifshitz points." 50

Let us pause here and contemplate the physical reasons for having such gapless modes. These excitations are not Goldstone bosons since neither of the Hamiltonians (5) and (7) possesses any continuous symmetry (which could be broken). Rather, these gapless modes appear as a result of "bottle-neck" quantum dynamics which only allows loops to fluctuate by either slowly growing or slowly shrinking: one lattice plaquette at a time. As a result we can think of configuration space as a very elongated essentially onedimensional object parametrized by the diameter of the biggest loop. While the quantum dynamics is in principle ergodic, in order to reach a state with a long order $L$ loop from a state with only short loops, the entire "length" of this "wormlike" graph has to be traversed. From this analogy, we see that the eigenvalue problem for our Hamiltonian is very similar to the eigenvalue problem for the Laplacian operator on a string. (In contrast, geometries such as a complete graph or hypercube do have spectral gaps; their bonds tie them together more efficiently than links in a linear chain.)

Now, what about the spectrum when the Jones-Wenzl projectors are implemented ${ }^{12}$ ? With the help of such additional terms, going from short to long loops can be achieved by merging existing loops together which can be done substantially faster than by "growing" them. These projectors would directly connect various points of the "wormlike" configuration space of the system. In the case $d=1$ adding the twostrand projector essentially reproduces Kitaev's "toric code $^{10}$ " and a gap is opened. (We expect this to be the case for $d=-1$ as well.) In the case of $n=d^{2}=2$, adding the threestrand projector is not sufficient because the probability of three long loops coming together within several lattice constants from each other-a necessary condition for a JW pro- 
jector to act efficiently_vanishes as $L^{-\alpha}$ for some $\alpha>0.51$ Hence the effect of such terms on the spectrum is too weak to open a gap (although it might lead to the "stiffening" of gapless excitations by reducing the dynamical exponent $z$ ).

\section{CONCLUSIONS}

In this paper, we have analyzed the spectrum of low-lying excitations for a general class of local Hamiltonians whose ground state(s) are characterized by $d$-isotopy. Using the statistical properties of their nonlocal degrees of freedom we established the analog of a Lieb-Schultz-Mattis theorem for quasitopological systems. The excitations are gapless with the variational ansatz strongly suggesting a quadratic $(\omega$ $\propto k^{2}$ ) dispersion. What may strike one as interesting and counter intuitive is the fact that both the Hamiltonian and the correlations of local operators are perfectly short-ranged, however, the quantum dynamics is constrained to operate on nonlocal quasi-long-ranged objects-loops - which in turn leads to a gapless spectrum. Finally, it is also interesting to remark on the potential implications of such a behavior from the perspective of understanding quantum glasses. The idea of using similar quasitopological models to model glassy behavior is not entirely new, ${ }^{52-54}$ but until very recently, ${ }^{55}$ all proposed models had a serious drawback, namely, quasilong-range correlations between local degrees of freedom. This is contrary to the fact that experimentally observed slow glassy dynamics has not been accompanied by any divergent correlations. In this paper we have explicitly demonstrated that such behavior is entirely possible in the context of quasitopological quantum critical points.

\section{ACKNOWLEDGMENTS}

The authors would like to thank Oded Schramm for illuminating discussions on the statistical properties of critical configurations. We are also thankful to Matthew Hastings for pointing out a gap in the proof presented in the earlier version of this manuscript. In addition, we would like to acknowledge the hospitality of KITP and the Aspen Center for Physics. C.N. and K.S. have been supported by the ARO under Grant No. W911NF-04-1-0236. C.N. has also been supported by the NSF under Grant No. DMR-0411800. *kirill.shtengel@ucr.edu

${ }^{1}$ X. G. Wen, Int. J. Mod. Phys. B 4, 239 (1990).

${ }^{2}$ S. Das Sarma and A. Pinczek, Perspectives in Quantum Hall Effects: Novel Quantum Liquids in Low-Dimensional Semiconductor Structures (Wiley, New York, 1997).

${ }^{3}$ P. W. Anderson, Science 235, 1196 (1987).

${ }^{4}$ S. A. Kivelson, D. S. Rokhsar, and J. P. Sethna, Phys. Rev. B 35, 8865 (1987)

${ }^{5}$ N. Read and S. Sachdev, Phys. Rev. Lett. 66, 1773 (1991).

${ }^{6}$ L. Balents, M. P. A. Fisher, and C. Nayak, Phys. Rev. B 61, 6307 (2000).

${ }^{7}$ T. Senthil and M. P. A. Fisher, Phys. Rev. B 62, 7850 (2000).

${ }^{8}$ R. B. Laughlin, Phys. Rev. Lett. 60, 2677 (1988).

${ }^{9}$ Y. Chen, F. Wilczek, E. Witten, and B. Halperin, Int. J. Mod. Phys. B 3, 1001 (1989).

${ }^{10}$ A. Y. Kitaev, Ann. Phys. (N.Y.) 303, 2 (2003).

${ }^{11}$ M. H. Freedman, M. J. Larsen, and Z. Wang, Commun. Math. Phys. 227, 605 (2002).

${ }^{12}$ M. Freedman, C. Nayak, K. Shtengel, K. Walker, and Z. Wang, Ann. Phys. (N.Y.) 310, 428 (2004).

${ }^{13}$ M. Freedman, C. Nayak, and K. Shtengel, arXiv:cond-mat/ 0309120 (unpublished)

${ }^{14}$ M. Freedman, C. Nayak, and K. Shtengel, Phys. Rev. Lett. 94, 147205 (2005).

${ }^{15}$ M. H. Freedman, Commun. Math. Phys. 234, 129 (2003).

${ }^{16}$ S. A. Trugman and S. Kivelson, Phys. Rev. B 31, 5280 (1985).

${ }^{17}$ F. D. M. Haldane, in The Quantum Hall Effect, 2nd ed., edited by R. E. Prange and S. M. Girvin (Springer-Verlag, New York, 1990), Chap. 8.

${ }^{18}$ C. K. Majumdar and D. K. Ghosh, J. Math. Phys. 10, 1388 (1969).

${ }^{19}$ D. J. Klein, J. Phys. A 15, 661 (1982).

${ }^{20}$ I. Affleck, T. Kennedy, E. H. Lieb, and H. Tasaki, Phys. Rev.
Lett. 59, 799 (1987).

${ }^{21}$ E. Lieb, T. Schultz, and D. Mattis, Ann. Phys. (N.Y.) 16, 407 (1961).

${ }^{22} \mathrm{~A}$ multiloop is a collection of nonintersecting loops and arcs on a surface. The end points of an arc are required to lie on the boundary of the surface, see Ref. 12.

${ }^{23}$ L. Kauffman and S. Lins, Temperley Lieb Recoupling Theory and Invariants of 3-Manifolds, Annals of Mathematics Studies Vol. 134 (Princeton University Press, Princeton, NJ, 1994).

${ }^{24}$ See Ref. 56 where private communication with A. Kitaev and G. Kuperberg is referenced. The required family of commuting projectors is easily derived in the Turaev-Viro approach (Ref. 57) by writing surface $\times$ interval, $\Sigma \times I=$ handle-body union 2-handles. The disjoint attaching curves of the 2-handles yield the commuting projectors.

${ }^{25}$ Here we use the term "frustration" in reference to a Hamiltonian which can be written as a sum of projectors yet has no zero modes. In this sense, new terms breaking the extensive degeneracy down to a crystalline structure will introduce frustration.

${ }^{26}$ R. B. Laughlin, Phys. Rev. Lett. 50, 1395 (1983).

${ }^{27} \mathrm{We}$ stress that this is a term-by-term correspondence in the sense that, given the appropriate basis, the squared amplitudes of the basis states comprising the ground state are identical to the Gibbs weights of the corresponding statistical mechanical states (up to an overall constant).

${ }^{28}$ D. S. Rokhsar and S. A. Kivelson, Phys. Rev. Lett. 61, 2376 (1988).

${ }^{29}$ R. Moessner and S. L. Sondhi, Phys. Rev. Lett. 86, 1881 (2001).

${ }^{30}$ G. Misguich, D. Serban, and V. Pasquier, Phys. Rev. Lett. 89, 137202 (2002).

${ }^{31}$ C. L. Henley, J. Stat. Phys. 89, 483 (1997).

${ }^{32}$ C. L. Henley, J. Phys.: Condens. Matter 16, S891 (2004).

${ }^{33}$ C. Castelnovo, C. Chamon, C. Mudry, and P. Pujol, Phys. Rev. B 
72, 104405 (2005).

${ }^{34}$ C. Castelnovo, C. Chamon, C. Mudry, and P. Pujol, Ann. Phys. (N.Y.) 318, 316 (2005).

${ }^{35}$ M. Freedman, C. Nayak, and K. Shtengel, Phys. Rev. Lett. 94, 066401 (2005).

${ }^{36}$ In a fully packed case, there is an additional geometric constraint limiting these winding numbers to either only even or only odd, depending on the size of the torus.

${ }^{37}$ C. M. Fortuin and P. W. Kasteleyn, Physica (Amsterdam) 57, 536 (1972).

${ }^{38}$ This derivation can be made slightly more formal if one defines a joint measure on both spin and bond configurations: $\mu(\sigma, \omega)$ $=v^{b(\omega)} \Delta(\sigma, \omega)$. This is known as Edwards-Sokal measure (Ref. 58). As follows from Eq. (12), when traced over both spins and bond occupations, it gives the desired partition function. Its marginal with respect to bond configurations is the Gibbs weight for spins, while its marginal with respect to spin configurations defines the weight for random clusters.

${ }^{39}$ It is also known as a cyclomatic number and for the case of a planar graph is equal to the number of finite faces (Ref. 59).

${ }^{40}$ R. J. Baxter, S. B. Kelland, and F. Y. Wu, J. Phys. A 9, 397 (1976).

${ }^{41}$ C. M. Fortuin, P. W. Kasteleyn, and J. Ginibre, Commun. Math. Phys. 22, 89 (1971).

${ }^{42}$ M. Aizenman, J. T. Chayes, L. Chayes, and C. M. Newman, J. Stat. Phys. 50, 1 (1988).

${ }^{43}$ G. R. Grimmett, Percolation, 2nd ed. (Springer-Verlag, Berlin,
1999).

${ }^{44}$ J. T. Chayes, A. L. Puha, and T. D. Sweet, in Probability Theory and Applications, IAS/Park City Mathematics Series Vol. 6, edited by E. Hsu and S. R. S. Varadhan (American Mathematical Society, Providence, 1999), pp. 49-166, http://www.cts.cuni.cz/ kotecky/seminars/spring/percnotes.ps

${ }^{45}$ E. Domany, D. Mukamel, B. Nienhuis, and A. Schwimmer, Nucl. Phys. 190, 279 (1981).

${ }^{46}$ B. Nienhuis, in Phase Transitions and Critical Phenomena, edited by C. Domb and J. L. Lebowitz (Academic, London, 1987), Vol. 11, pp. 1-53.

${ }^{47}$ V. G. Turaev and O. Y. Viro, Topology 31, 865 (1992).

${ }^{48}$ M. B. Hastings, Phys. Rev. B 69, 104431 (2004).

${ }^{49}$ M. B. Hastings, Europhys. Lett. 70, 824 (2005).

${ }^{50}$ E. Ardonne, P. Fendley, and E. Fradkin, Ann. Phys. (N.Y.) 310, 493 (2004)

${ }^{51}$ O. Schramm (private communication)

${ }^{52}$ H. Yin and B. Chakraborty, Phys. Rev. Lett. 86, 2058 (2001).

${ }^{53}$ H. Yin and B. Chakraborty, Phys. Rev. E 65, 036119 (2002).

${ }^{54}$ D. Das, J. Kondev, and B. Chakraborty, arXiv:cond-mat/ 0112281 (unpublished).

${ }^{55}$ C. Chamon, Phys. Rev. Lett. 94, 040402 (2005).

${ }^{56}$ M. H. Freedman, Found Comput. Math. 1, 183 (2001).

${ }^{57}$ V. G. Turaev, Quantum Invariants of Knots and 3-Manifolds (Walter de Gruyter, Berlin, 1994).

${ }^{58}$ R. G. Edwards and A. D. Sokal, Phys. Rev. D 38, 2009 (1988).

${ }^{59}$ J. W. Essam and M. E. Fisher, Rev. Mod. Phys. 42, 272 (1970). 